\title{
Magnetic Resonance Imaging Characterization of the Hippocampi in Temporal Lobe Epilepsy: Correlation of Volumetry and Apparent Diffusion Coefficient with Laterality and Duration of Seizures
}

\author{
Apoorva Muralidhar ${ }^{1} \quad$ Ashok Kumar ${ }^{1} \quad$ Arjun Prakash ${ }^{2} \quad$ Umesh Krishnamurthy ${ }^{1}$ Manjunath $\mathrm{S}^{1}$ \\ Roshni Majeed ${ }^{1}$ \\ ${ }^{1}$ Department of Radio-diagnosis, M. S. Ramaiah Medical College, \\ Bengaluru, Karnataka, India \\ 2Department of Radio-diagnosis, Bangalore Medical College \& \\ Research Institute, Bengaluru, Karnataka, India

\begin{abstract}
Address for correspondence Arjun Prakash, MD, DNB, Department of Radio-diagnosis, Bangalore Medical College \& Research Institute, Fort, KR Road, Bengaluru - 560 002, Karnataka, India (e-mail: drarjunprakash@gmail.com).
\end{abstract}

Indian J Radiol Imaging 2021;31:109-115.

\begin{abstract}
Keywords

- apparent diffusion coefficient

- hippocampal atrophy

- hippocampal volume

- volumetry

- mesial temporal sclerosis

- temporal lobe epilepsy
\end{abstract}

Background and Purpose It is estimated that hippocampal damage is seen in 50 to $70 \%$ of patients with temporal lobe epilepsy (TLE). Although most magnetic resonance imaging (MRI) studies are adequate to detect gross hippocampal atrophy, subtle changes that may characterize early disease in TLE, such as visually nonappreciable volume loss, may often be missed if objective volumetric analysis is not undertaken.

Materials and Methods We conducted a hospital-based prospective analytical study in which 40 patients with partial seizures of temporal lobe origin were included and their hippocampal volumes (HVs) were determined by manual volumetric analysis. The findings were recorded and correlated with the side of seizure and its duration. The quantitative assessment was allotted different grades accordingly. Also, the apparent diffusion coefficient (ADC) values of bilateral hippocampi were estimated and their correlation with the side of seizure was determined.

Results Most patients in the study were in the age group of 11 to 20 years (37.5\%). In total, $57.5 \%$ had seizures for a period of 1 to 5 years. While $67.5 \%(n=27)$ had seizure on the right, $32.5 \%(n=13)$ had on the left. The mean HV estimated on the right and left were correlated with the side of seizure and found to be statistically significant $(p<$ 0.001 in those with right-sided seizures and $p=0.02$ in those with left-sided seizures). Simultaneously the ADC values estimated were found to correlate with the laterality of seizures with a statistical difference $(p<0.01)$. Duration of seizures however did not show a positive correlation with the HV.

Conclusion MRI with quantitative estimation of HV and ADC values can depict the presence and laterality in TLE with accuracy rates that exceed those achieved by visual inspection alone. Thus, quantitative MRI provides a useful means for translating volumetric analysis into clinical practice. published online May 31, 2021
DOI https://doi.org/

$10.1055 / \mathrm{s}-0041-1729672$

ISSN 0971-3026
C 2021 . Indian Radiological Association
This is an open access article published by Thieme under the terms of the Creative
Commons Attribution-NonDerivative-NonCommercial-License, permitting copying
and reproduction so long as the original work is given appropriate credit. Contents
may not be used for commercial purposes, or adapted, remixed, transformed or
built upon. (https://creativecommons.org/licenses/by-nc-nd/4.0/).
Thieme Medical and Scientific Publishers Private Ltd. A-12, Second
Floor, Sector -2, NOIDA - 201301 , India 


\section{Introduction}

Mesial temporal lobe epilepsy (MTLE) is the most common syndrome associated with focal seizures with dyscognitive features and characteristic clinical, electroencephalographic (EEG), and pathologic features. ${ }^{1}$ Synonyms for this entity include "psychomotor seizures," "limbic seizures," and "temporal lobe seizures." It is said to be "complex partial" if there is loss of awareness or "simple partial" if awareness is retained.

Magnetic resonance imaging (MRI) can detect the characteristic hippocampal sclerosis (HS) that is an important feature in patients with MTLE. Also, these patients have hippocampal atrophy (HA) of varying severity and degree. However, the pathogenesis of HS is not well understood. Various theories and postulates put forth include ischemia/hypoxia, neurodegeneration, or a combination of these. ${ }^{2}$

While gross HA is detected with ease on MRI studies, subtle HA that may characterize early disease in MTLE such as visually nonappreciable volume loss may often be missed if objective volumetric analysis is not done. Hippocampal volume (HV) is a strong surrogate marker for the presence and severity of HA., ${ }^{3,4}$ The present study was an attempt to facilitate the interpretation of HA by quantitative volumetric analysis bilaterally and the apparent diffusion coefficient (ADC) values and in turn determine if the side of seizure activity and duration of seizures correlate with these entities. Such quantitative assessment helps in establishing early and accurate diagnosis.

\section{Materials and Methods}

\section{Patient Selection}

After approval from the institutional ethics review committee, a hospital-based prospective study was conducted. The study group composed of 40 patients presenting to the Departments of Radio-diagnosis, Neurology, Neurosurgery, and Medicine with focal seizures with or without secondary generalization, undergoing MRI of brain in a tertiary care center of South India for a period of 2 years. Those patients with focal seizures attributed to space-occupying lesion in the brain and those with prior history of surgery to the temporal lobes were excluded from the study. Informed and written consent were obtained from all the 40 participants.

\section{Aims and Objectives}

1. To estimate the volume of hippocampi bilaterally in patients with temporal lobe epilepsy and determine the relation of $\mathrm{HV}$ with the duration and frequency of seizures.

2. To calculate the ADC value of hippocampi bilaterally.

3. To determine whether the volume and ADC values are related to the side of seizure activity.

\section{MRI Protocol}

Using 1.5 Tesla (Siemens Magnetom Avanto, Germany) and a standard head coil, T1 Gradient Echo sequences (MPRAGE TR: $19.2 \mathrm{~ms}$, TE: $3.5 \mathrm{~ms}$, TI: $11 \mathrm{~ms}$, flip angle: 15 degrees, matrix: $92 \times 256$ images of $1 \mathrm{~mm}$ slice thickness) were obtained perpendicular to the long axis of the hippocampus as shown in - Fig. 1. T2-weighted images and FLAIR oblique coronal sequences were used to assess signal change. Axial $\mathrm{ADC}$ maps were used for ADC value estimation.

\section{Image Analysis}

The study was double blinded and estimation of HV and ADC were done independently by two radiologists, including a radiologist with more than 10 years of experience. The HV including volume of dentate gyrus, hippocampus proper, and subiculum complex were manually delineated on an average of 40 slices bilaterally. The anterior boundary was taken as the first slice where hippocampus was visible and the posterior border was the slice before total length of fornix was visible. The lateral boundaries were defined by the temporal horn of lateral ventricle and gray-white matter border of temporal stem and medially by the cisterna ambiens and transverse fissure. Ventrally it was limited by the parahippocampal gyrus.

Also, MRIs were evaluated for volume loss visually and high signal intensity on T2-weighted images. Secondary signs of HA-like atrophy of the amygdala, mammillary body, and fornices were also assessed. Medial temporal lobe atrophy (MTA) scores were allotted on the right and left sides for each patient as given in - Table 1. ADC values were measured in the hippocampal region on axial sections with 1.5 to $2.5 \mathrm{~cm}^{3}$ region of interest (ROI) and containing 150 to 250 voxels bilaterally for all patients.

\section{Statistical Analysis}

The relationship between clinical lateralization of seizure, $\mathrm{HV}$, and ADCs on the right and left sides were analyzed using SPSS software version 18.0 (IBM, Somers, New York, United States). Data were presented in terms of percentage and mean. Student's $t$-test was used to estimate the $p$-value and significance. Pearson's correlation was used to correlate HV and the duration of seizure.

\section{Results}

\section{Patient Characteristics}

There were 24 male (60\%) and 16 female (40\%) patients in our study. The age group ranged from 10 years to 72 years with a mean age of 29 years. Most patients were in the second decade with a male predominance. This is explained in - Table 2.

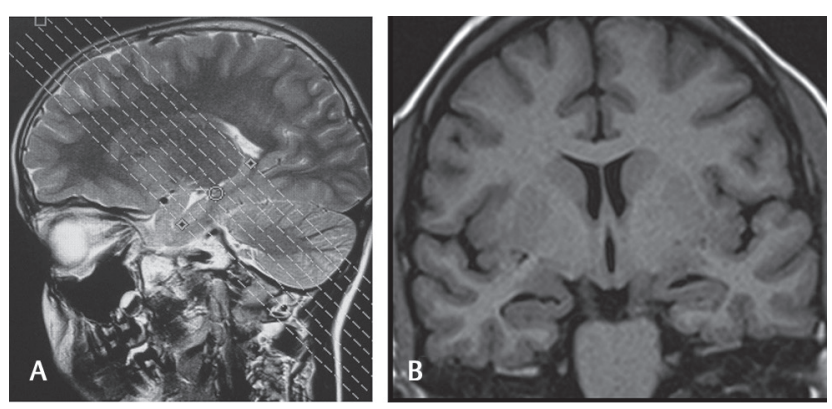

Fig. 1 (A, B) Planning along the long axis of hippocampus on sagittal image (A) to obtain coronal oblique T1IR image (B). 
Table 1 Assessment of MTA scores based on three parameters-width of choroid fissure, width of temporal horn, and height of the hippocampus

\begin{tabular}{|l|l|l|l|}
\hline \multicolumn{4}{|c|}{ MTA visual rating scale } \\
\hline Score & Width of choroid fissure & Width of temporal horn & Height of hippocampal formation \\
\hline 0 & $\mathrm{~N}$ & $\mathrm{~N}$ & $\mathrm{~N}$ \\
\hline 1 & $\uparrow$ & $\mathrm{N}$ & $\mathrm{N}$ \\
\hline 2 & $\uparrow \uparrow$ & $\uparrow \uparrow$ & $\downarrow$ \\
\hline 3 & $\uparrow \uparrow \uparrow$ & $\uparrow \uparrow \uparrow$ & $\downarrow \downarrow$ \\
\hline 4 & $\uparrow \uparrow \uparrow$ & $\uparrow \uparrow \uparrow$ & $\downarrow \downarrow \downarrow$ \\
\hline
\end{tabular}

Abbreviation: MTA, medial temporal lobe atrophy.

Table 2 In this study, peak incidence of temporal lobe epilepsy occurred in the age group of 11-20 years in both male and female patients accounting for $37.5 \%$ each

\begin{tabular}{|l|l|l|l|l|}
\hline Age $(\mathrm{y})$ & Frequency of males & Percentage & Frequency of females & Percentage \\
\hline $1-10$ & 2 & 8.4 & 1 & 6.25 \\
\hline $11-20$ & 9 & 37.5 & 6 & 37.5 \\
\hline $21-30$ & 5 & 20.9 & 5 & 31.25 \\
\hline $31-40$ & 1 & 4.1 & 1 & 6.25 \\
\hline $41-50$ & 1 & 4.1 & 1 & 6.25 \\
\hline$>50$ & 6 & 25 & 2 & 12.5 \\
\hline Total & 24 & 100 & 16 & 100 \\
\hline
\end{tabular}

\section{Duration of Seizures}

Most patients (57.5\%, $n=23$ ) had seizures for a duration of 1 to 5 years. A total of $30 \%(n=12)$ had seizures from 5 to 10 years. Only one patient had seizures for more than 10 years ( - Fig. 2 ).

\section{Laterality of Seizures}

A total of $67.5 \%(n=27)$ patients had focal seizures on the right and the remaining $37.5 \%(n=13)$ on the left. Among these patients, approximately $40 \%$ had focal seizures with secondary generalization. The side was determined primarily based on history, with supporting evidence of epileptic spikes on EEG wherever available.

\section{Volume of Hippocampi}

Visual assessment of the hippocampi revealed asymmetry on both sides in 55\% $(n=22)$ and symmetricity in $45 \%(n=18)$. The internal architecture and gray-white matter differentiation were lost in those with severe atrophy with significant reduction in size in $40 \%$. The dilatation of temporal horn of lateral ventricle, width of choroid fissure, and hippocampal height was evaluated in each patient and graded into groups based on the MTA score. Those with right focal seizures had almost equidistribution in all the groups with 22.5, 27.5, 20, 17.5 , and $12.5 \%$ respectively with a score of $0,1,2,3$, and 4 . Those with left focal seizures, however, showed majority in the score 0 group with $42.5 \%$. A total of $22.5 \%$ had a score of 1 , $15 \%$ had a score of $2,12.5 \%$ had a score of 3 , and $7.5 \%$ a score of 4 (-Fig. 3).

Absolute value of the volume of bilateral hippocampi was determined by manual tracing in consecutive sections

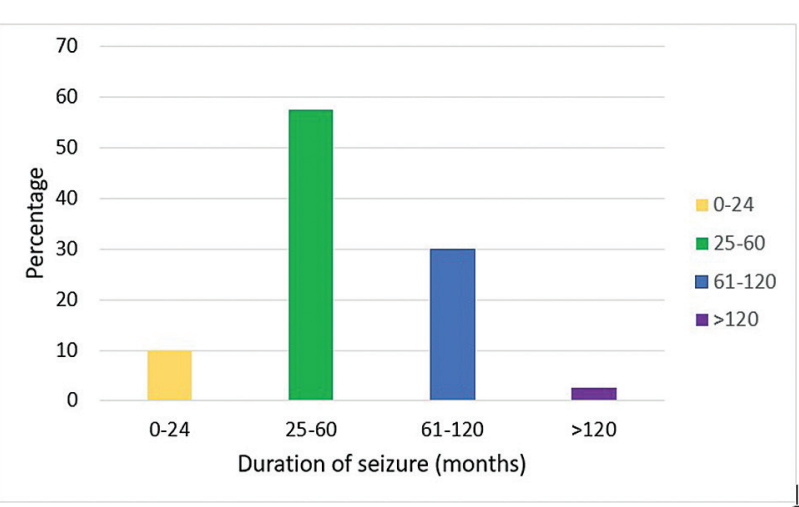

Fig. 2 Duration of seizures. In this study, most patients (57.5\%) had seizures for a duration of 25-60 months, the next highest being $33 \%$ with a duration of $60-120$ months.

(-Fig. 4) and found to have reductions in patients with TLE in $65 \%(n=26)$ either on the right or left or bilaterally. Few examples where differences in hippocampal volume on both sides were estimated by volumetry are shown in -Figs. 5 and 6. The least measured volume was of a 24-year-old female measuring $1,670 \mathrm{~mm}^{3}$ on the right and $1,730 \mathrm{~mm}^{3}$ on the left. At the other end of the spectrum was a 15-year-old male with normal HV without evidence of atrophy. The volume on the right was $3,210 \mathrm{~mm}^{3}$ and on the left was $3420 \mathrm{~mm}^{3}$.

As evidenced in - Fig. 7, of the 27 patients with right-sided seizure, the mean $\mathrm{HV}$ on the right was $2,367 \mathrm{~mm}^{3}$ and on the contralateral side was $2,763 \mathrm{~mm}^{3}$. Of the 13 patients with left-sided seizure, the mean $\mathrm{HV}$ on the right was $2,860 \mathrm{~mm}^{3}$ and on the ipsilateral side was $2,552 \mathrm{~mm}^{3}$. 
A

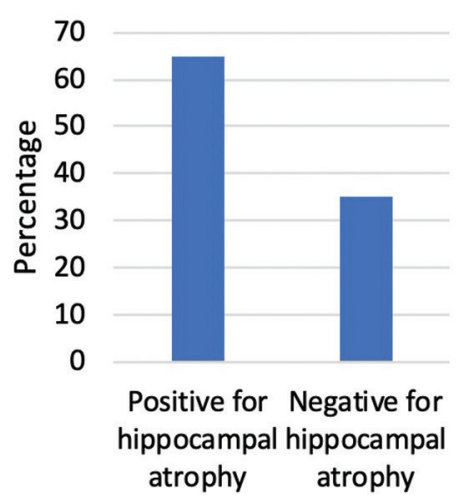

B

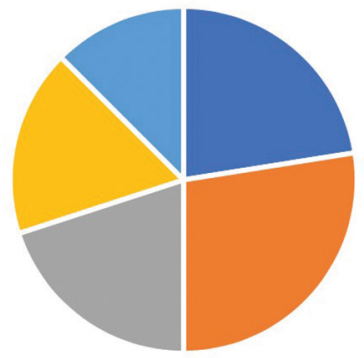

$\because 0=1=2\|3\| 4=5$
C Left

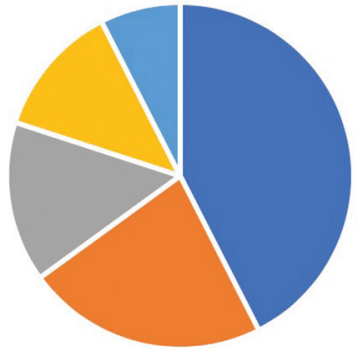

$=0-1=2-3=4=5$

Fig. 3 (A-C) Hippocampal atrophy in temporal lobe epilepsy patients. (A) In this study, of the 40 patients, 26 had hippocampal atrophy (65\%) of varying grades either on the right or left. (B) MTA score of $0,1,2,3$, and 4 were seen in $22.5,27.5,20,17.5$, and $12.5 \%$, respectively, on the right. (C) MTA scores of $0,1,2,3$, and 4 were seen in $42.5,22.5,15,12.5$, and $7.5 \%$, respectively, on the left. MTA, medial temporal lobe atrophy.
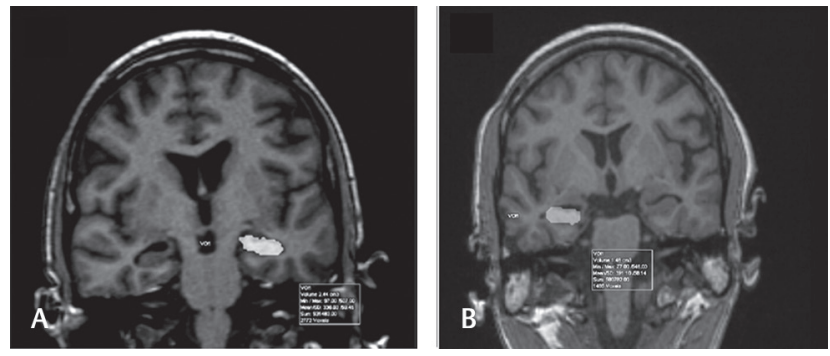

Fig. 4 (A, B) Volume estimation. (A) Coronal 3D-MP-RAGE image demonstrating the method to draw volume of interest (VOI) for estimation of hippocampal volume. In this patient with right-sided seizure, volume of the left hippocampus was estimated to be $2.44 \mathrm{~cm}^{3}$ while (B) on the right was $1.48 \mathrm{~cm}^{3}$.
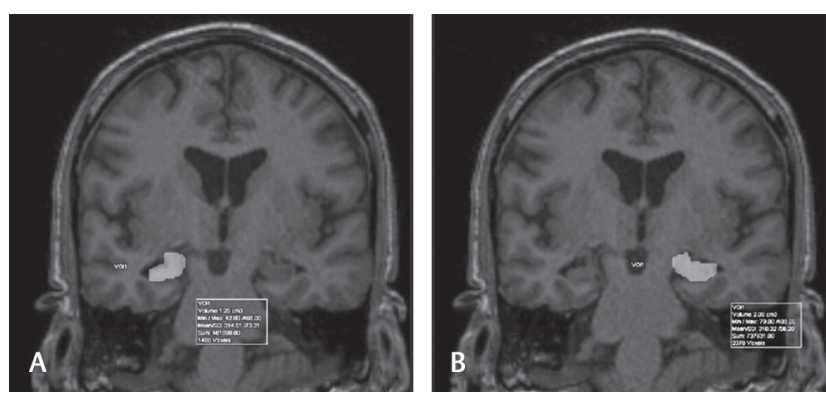

Fig. 6 (A, B) Volume estimation. A patient with right side focal seizures with evidence of significant right hippocampal atrophy. (A) The right hippocampal volume was estimated to be $1.28 \mathrm{~cm}^{3}$ while (B) on the left was $2.06 \mathrm{~cm}^{3}$.

These values were compared with the control values studied in the Indian population. HV correlated with the laterality of seizure onset with statistical significance, $p<0.001$ was seen in those with right-sided seizures and $p=0.02$ in those with left-sided seizures.

Correlation of the HV on both sides with duration of seizures did not show a positive correlation using Pearson's
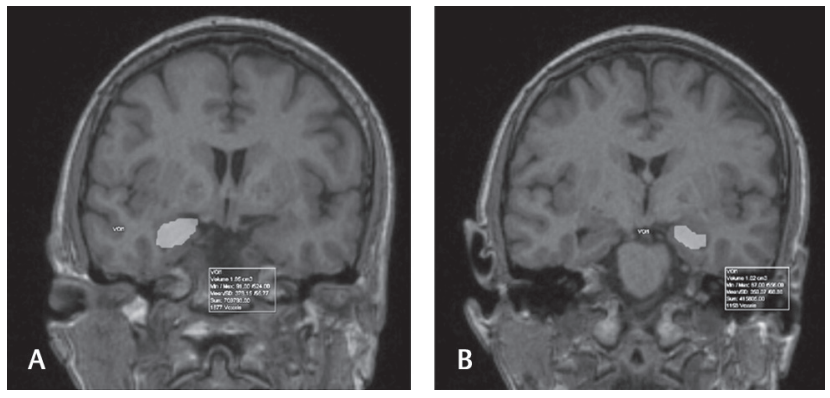

Fig. 5 (A, B) Volume estimation. A patient with left side focal seizures with evidence of significant bilateral hippocampal atrophy. (A) The right hippocampal volume was estimated to be $1.65 \mathrm{~cm}^{3}$ while (B) on the left was $1.02 \mathrm{~cm}^{3}$.
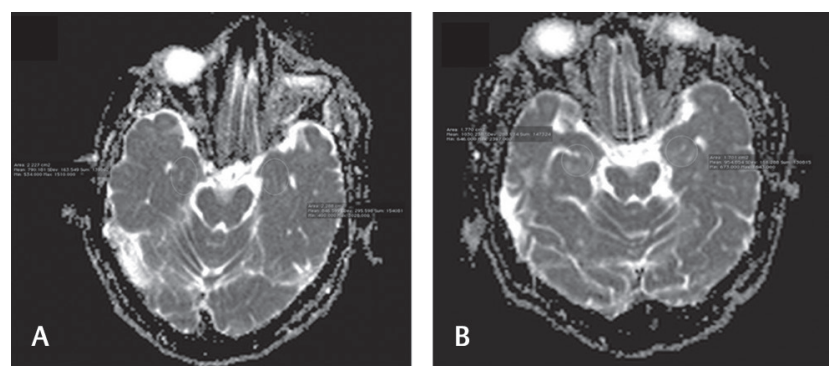

Fig. 7 (A, B) Mean hippocampal volume (HV) in right- and left-sided seizures. (A) Of the 27 patients with right-sided seizures, the mean $\mathrm{HV}$ on the right is $2,367 \mathrm{~mm}^{3}$ and on the left is $2,763 \mathrm{~mm}^{3}$. (B) Of the 13 patients with left-sided seizure, the mean $\mathrm{HV}$ on the right is $2,860 \mathrm{~mm}^{3}$ and on the left is $2,553 \mathrm{~mm}^{3}$.

correlation. The reason is likely due to several confounding factors such as frequency of seizures, intensity or severity of seizures, age of presentation, and most importantly treatment and compliance with antiepileptic drugs (-Fig. 8). Mammillary body and fornix atrophy were present in 60\% while that of amygdala atrophy was seen in $70 \%$ of patients with MTA scores of 3 and 4. 


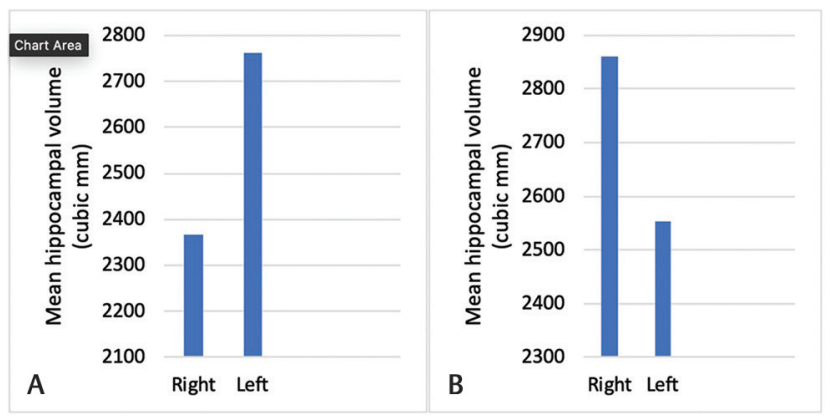

Fig. 8 (A, B) Pearson's correlation is used to correlate HV and the duration of seizure. The correlation coefficient " $r$ " is 0.57 on the right (A) and 0.55 on the left (B). HV, hippocampal volume.

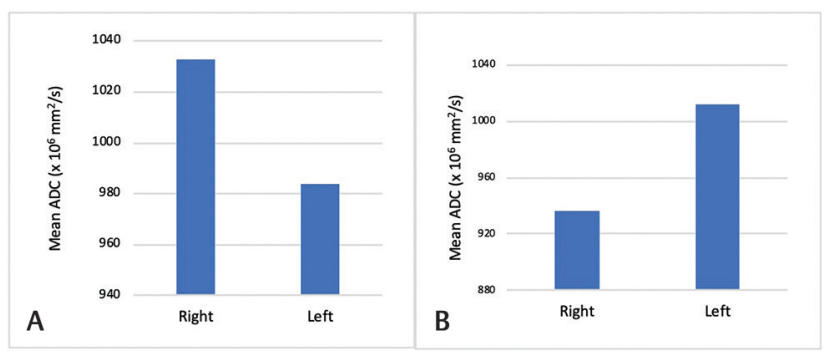

Fig. 10 (A, B) Mean ADC values in right- and left-sided seizures. (A) Of the 27 patients with right-sided seizure, the mean ADC value on the right is $1,033 \times 10^{-6} \mathrm{~mm}^{2} / \mathrm{s}$ and on the left is $984 \times 10^{-6} \mathrm{~mm}^{2} / \mathrm{s}$. (B) Of the 13 patients with left-sided seizure, the mean ADC value on the right is $936 \times 10^{-6} \mathrm{~mm}^{2} / \mathrm{s}$ and on the left is $1012 \times 10^{-6} \mathrm{~mm}^{2} / \mathrm{s}$. $A D C$, apparent diffusion coefficient.

\section{Evaluation of ADC}

ADC values were measured in the hippocampal region on axial sections with 1.5 to $2.5 \mathrm{~cm}^{3}$ ROI and containing 150 to 250 voxels bilaterally for all patients ( $\boldsymbol{- F i g . 9}$ ). Of the 27 patients with right-sided seizure, the mean ADC value on the right was $1,033 \times 10^{-6} \mathrm{~mm}^{2} / \mathrm{s}$ and on the left was $984 \times$ $10^{-6} \mathrm{~mm}^{2} / \mathrm{s}$. Of the 13 patients with left-sided seizure, the mean ADC value on the right was $936 \times 10^{-6} \mathrm{~mm}^{2} / \mathrm{s}$ and on the left was $1012 \times 10^{-6} \mathrm{~mm}^{2} / \mathrm{s}$ ( - Fig. 10). These ADC values obtained were found to aid in the determination of the laterality of seizure, even in the absence of visually apparent differences in ADC. Statistical significance in predicting the laterality based on the ADC values on the right and left was found to be $p=0.04$ in those with right-sided seizure and $p=0.01$ in those with left-sided seizure.

\section{Discussion}

TLE was defined by the International League Against Epilepsy as a condition characterized by recurrent, unprovoked seizures originating from the medial or lateral temporal lobe. Mesial temporal sclerosis (MTS), also commonly referred to as HS, is the most common association with intractable TLE presenting as partial complex seizures. The pathophysiology of MTS is not entirely understood. One theory is that early or prolonged febrile seizures damage the hippocampus in genetically susceptible patients. The risk factors for TLE include head trauma with loss of consciousness, injuries
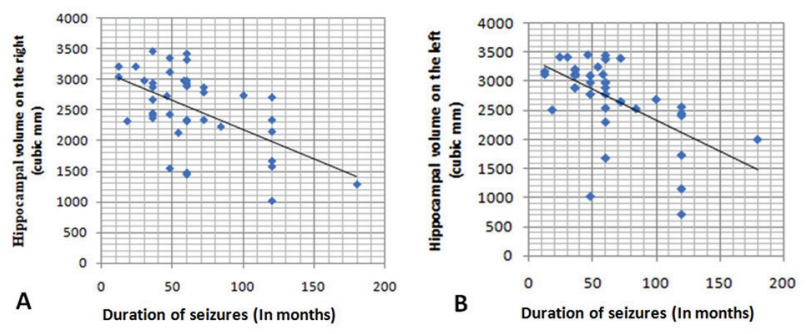

Fig. 9 (A, B) ADC estimation. Axial ADC image showing method to estimate $A D C$ values of bilateral hippocampi. (A) A 55-year-old male with focal seizure on the left showed increased ADC on the left side. (B) A 20-year-old female with focal seizure on the right showed increased $A D C$ on the right side. $A D C$, apparent diffusion coefficient.

during early childhood and birth, brain malformations, infections such as encephalitis or meningitis, and tumors in the temporal lobe. MTLE may have onset at any age, though most commonly it occurs in the later first or second decade. The seizures may be associated with memory impairment, aura, somatosensory and special sensory (olfactory, gustatory, and visual) illusions and hallucinations, vertigo, and autonomic changes. ${ }^{5}$

The diagnosis is based on clinical history of the seizure episode. EEG shows anterior temporal spike or sharp waves. MRI of the brain is the standard imaging modality for assessment of HS. Neuronal cell loss and gliosis result in increased T2/FLAIR signal intensity. Other secondary signs include loss of the internal architecture of the hippocampus, loss of hippocampal head digitations, dilatation of the ipsilateral temporal horn, increased signal intensity, and/or atrophy of the ipsilateral amygdala, mammillary body and fornix..$^{6-8} \mathrm{HV}$ may vary based on ethnicity and genetics. Although results obtained from studies on Western counterparts were scrutinized, this study primarily focused on values obtained from Indian data as control. ${ }^{9-11}$ The anatomic boundaries for estimation of the HV have been discussed earlier and volumes hence calculated on both sides were compared with the laterality of the seizure onset. ${ }^{12,13}$

MTA score is a visual score performed on coronal T1-weighted images through the hippocampus at the level of the anterior pons and assesses the width of the choroid fissure, width of the temporal horn of the lateral ventricle, and height of the hippocampi. ${ }^{14}$

In a study by Mohandas et al,,$^{15}$ a mean HV of $2.411 \mathrm{~cm}^{3}$ (standard deviation 0.299) was found, which was significantly smaller when compared with the data from the western population. The right hippocampus was larger than the left, with a mean volume of 2.424 and $2.398 \mathrm{~cm}^{3}$, respectively.

In a study conducted by Hakyemez et $\mathrm{al}^{16}$ there was HA in $84 \%$ and increased signal intensity in $60 \%$, unilateral atrophy in $88 \%$, and bilateral atrophy in $13 \%$. While in our study, $65 \%$ had HA, of which $67.5 \%$ had on the right side while $37.5 \%$ had on the left side.

In a study by García-Fiñana et al, ${ }^{17}$ it was proposed that both right and left HVs were on an average significantly smaller in patients with right-sided seizure onset and left HV was smaller in patients with left-sided seizure. In our study, we obtained similar results. 
Various hypotheses have been put forth to explain changes in ADC in epilepsy. A higher ADC value in sclerotic hippocampus is said to result due to relative increase of interstitial water proton secondary to neuronal cell loss and/or gliosis. The initial ADC decrease is associated with neuronal swelling and subsequent increase in $A D C$ is due to microstructural damage with necrosis, gliosis, and expanded extracellular space. Another explanation for increased ADC in sclerotic hippocampi is partial volume averaging artifact because of more cerebrospinal fluid space included in the ROI in sclerotic hippocampi.

In a study by Londoño et $a{ }^{18}{ }^{18}$ it was estimated that the mean ADC value of the contralateral side or the normal hippocampus in patients with TLE was also significantly higher than in healthy people. They also concluded that quantitative measurement of $A D C$ enabled correct lateralization of the affected side in $100 \%$. A normal ADC value in a visually abnormal hippocampus may be the presence of concomitant acute and chronic changes. Decreased ADC values have been observed after status epilepticus. ${ }^{19}$

In our study, higher mean ADC values were seen on the side of seizure activity, similar to that seen in earlier studies.

TLE is initially managed medically with antiepileptic agents. In patients who are refractory to medical management, temporal lobectomy or selective amygdalohippocampectomy may be performed. Anterior temporal lobectomy may also be considered in a subgroup of patients with MTS with a success rate of 75 to $90 \% .^{20,21}$

\section{Limitations of the Study}

In spite of best efforts to keep the fallacies to a minimum, there are a few inadequacies in the study. The brief period of study and inability to follow up the cases for a prolonged period were inherent limitations. Visual grading of the hippocampi and mammillary bodies is quite subjective with some degree of overlap among the grades, contributing to interobserver variations. The ADC measurements of hippocampi were done on the axial plane, which might lead to an increased partial volume averaging artifact in contrast to measurement in the coronal plane, which is ideal for hippocampal evaluation.

\section{Conclusion}

$\mathrm{HV}$ on the ipsilateral side of seizure is significantly reduced in TLE. The duration of seizures is not linearly correlative with HV reduction due to possible other confounding factors such as treatment and frequency or severity of seizures, which are difficult to objectively determine with surety. ADC values are a useful means for determining HS in patients with TLE and abnormal values help determine laterality of seizure onset. Even when the visual assessment appears normal, secondary signs like atrophy of mammillary body and forniceal thinning are important clues to evaluate further. Combining results of volumetry and ADC maps gives superior results than either alone. The ADC value may be abnormal in the presence of normal magnetic resonance appearance and therefore is a valuable indicator in the initial detection of $\mathrm{HS}$.

\section{Declaration of Patient Consent}

The authors certify that they have obtained all appropriate patient consent forms. In the form the patient(s) has/have given his/her/their consent for his/her/their images and other clinical information to be reported in the journal. The patients understand that their names and initials will not be published and due efforts will be made to conceal their identity, but anonymity cannot be guaranteed.

\section{Financial Support and Sponsorship}

Nil.

\section{Conflicts of Interest}

There are no conflicts of interest.

\section{References}

1 Engel J Jr. Report of the ILAE classification core group. Epilepsia 2006;47(9):1558-1568

2 French JA, Williamson PD, Thadani VM, et al. Characteristics of medial temporal lobe epilepsy: I. Results of history and physical examination. Ann Neurol 1993;34(6):774-780

3 Kobayashi E, D’Agostino MD, Lopes-Cendes I, et al. Hippocampal atrophy and T2-weighted signal changes in familial mesial temporal lobe epilepsy. Neurology 2003;60(3):405-409

4 Zarow C, Wang L, Chui HC, Weiner MW, Csernansky JG. MRI shows more severe hippocampal atrophy and shape deformation in hippocampal sclerosis than in Alzheimer's disease. Int J Alzheimers Dis 2011;2011:483972

5 Lüders H, Acharya J, Baumgartner C, et al. Semiological seizure classification. Epilepsia 1998;39(9):1006-1013

6 Quigg M, Bertram EH, Jackson T, Laws E. Volumetric magnetic resonance imaging evidence of bilateral hippocampal atrophy in mesial temporal lobe epilepsy. Epilepsia 1997;38(5):588-594

7 Jackson GD, Kuzniecky RI, Cascino GD. Hippocampal sclerosis without detectable hippocampal atrophy. Neurology 1994;44(1):42-46

8 Kim JA, Chung JI, Yoon PH, et al. Transient MR signal changes in patients with generalized tonicoclonic seizure or status epilepticus: periictal diffusion-weighted imaging. AJNR Am J Neuroradiol 2001;22(6):1149-1160

9 Honeycutt NA, Smith CD. Hippocampal volume measurements using magnetic resonance imaging in normal young adults. J Neuroimaging 1995;5(2):95-100

10 Bhatia S, Bookheimer SY, Gaillard WD, Theodore WH. Measurement of whole temporal lobe and hippocampus for MR volumetry: normative data. Neurology 1993;43(10):2006-2010

11 Dhikav V, Duraisamy S, Anand KS, Garga UC. Hippocampal volumes among older Indian adults: comparison with Alzheimer's disease and mild cognitive impairment. Ann Indian Acad Neurol 2016;19(2):195-200

12 Jack CR Jr, Theodore WH, Cook M, McCarthy G. MRI-based hippocampal volumetrics: data acquisition, normal ranges, and optimal protocol. Magn Reson Imaging 1995;13(8):1057-1064

13 Jack CR Jr, Twomey CK, Zinsmeister AR, Sharbrough FW, Petersen RC, Cascino GD. Anterior temporal lobes and hippocampal formations: normative volumetric measurements from MR images in young adults. Radiology 1989;172(2):549-554

14 Wahlund LO, Julin P, Johansson SE, Scheltens P. Visual rating and volumetry of the medial temporal lobe on magnetic resonance imaging in dementia: a comparative study. J Neurol Neurosurg Psychiatry 2000;69(5):630-635

15 Mohandas AN, Bharath RD, Prathyusha PV, Gupta AK. Hippocampal volumetry: normative data in the Indian population. Ann Indian Acad Neurol 2014;17(3):267-271 
16 Hakyemez B, Yucel K, Yildirim N, Erdogan C, Bora I, Parlak M. Morphologic and volumetric analysis of amygdala, hippocampus, fornix and mamillary body with MRI in patients with temporal lobe epilepsy. Neuroradiol J 2006;19(3):289-296

17 García-Fiñana M, Denby CE, Keller SS, Wieshmann UC, Roberts N. Degree of hippocampal atrophy is related to side of seizure onset in temporal lobe epilepsy. AJNR Am J Neuroradiol 2006;27(5):1046-1052

18 Londoño A, Castillo M, Lee YZ, Smith JK. Apparent diffusion coefficient measurements in the hippocampi in patients with temporal lobe seizures. AJNR Am J Neuroradiol 2003;24(8):1582-1586
19 Yoo SY, Chang KH, Song IC, et al. Apparent diffusion coefficient value of the hippocampus in patients with hippocampal sclerosis and in healthy volunteers. AJNR Am J Neuroradiol 2002;23(5):809-812

20 Diehl B, Najm I, Ruggieri P, et al. Postictal diffusion-weighted imaging for the localization of focal epileptic areas in temporal lobe epilepsy. Epilepsia 2001;42(1):21-28

21 Ramey WL, Martirosyan NL, Lieu CM. Hasham HA, Lemole GM Jr, Weinand ME. Current management and surgical outcomes of medically intractable epilepsy. Clin Neurol Neurosurg 2013;115(12):2411-2418 